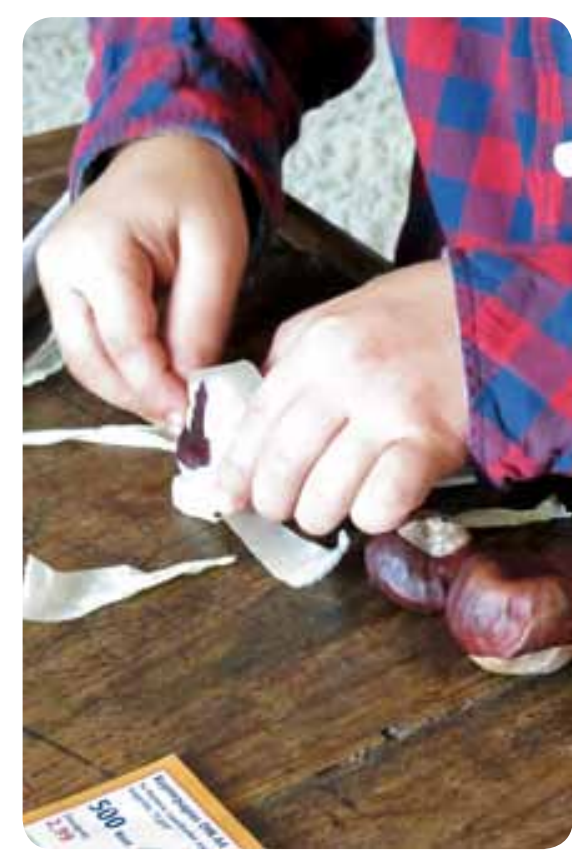

Abb.4-5: Spielen ist ein kreativer Prozess

versteckt sind. Lustvoll werden die Kastanien herausgesucht und wenig später mit Klebeband eingewickelt. Am Ende haben alle den verregneten Nachmittag

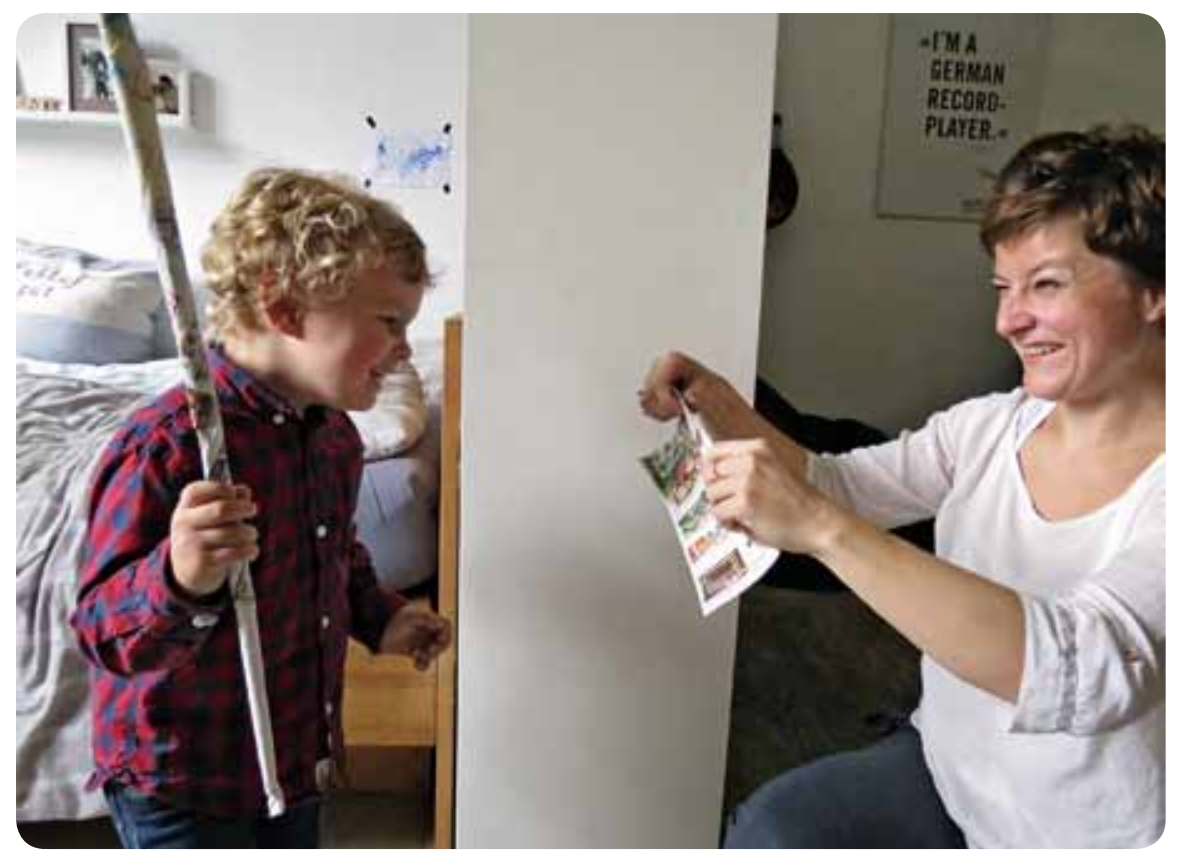

im wahrsten Sinne des Wortes sinnvoll gefüllt mit wenig Alltagsmaterial und

Anika Krüger

DOI 10.2378/motorik2016.art16d

viel Kreativität (Abb.4-5).

\title{
Praxisbeispiele für ein generationsübergreifendes Psychomotorikangebot
}

Aus der psychomotorischen Literatur wurden Spiele aus den Feldern psychomotorische Entwicklungsbegleitung mit Kindern, Motogeragogik (nach Philippi-Eisenburger bzw. Eisenburger 1998; 1999; 2012) und ganzheitliches Gehirntraining für Seniorlnnen (Schmidt/Schießl 1999) miteinander verglichen und ähnliche Spielformen herausgearbeitet. Die Intention dabei war, dass Spiele kindgerecht sein sollen, ohne kindlich zu wirken. Die meisten Spiele finden im Stuhlkreis statt, da die Gruppe der SeniorInnen in ihrer
Bewegung beeinträchtig ist. Die Spiele stellen erste Versuche für die praktische Arbeit mit zwei unterschiedlichen Generationen im psychomotorischen Kontext dar.

\section{Spielideen}

Folgende Spielideen kamen in der Gruppe positiv an, da die beiden Generationen sich bei diesen Spielen ohne Leistungsdruck kreativ ausagieren können:

\section{Murmelkunst im Schuhkarton}

Der Anleitende lässt ein Säckchen mit Murmeln im Kreis herumgehen. Die Teilnehmenden erfühlen den Inhalt. Danach verteilt der Anleiter an jedes Kind und jeden/jede Seniorln einen Schuhkartondeckel. Im Anschluss werden die Murmeln mit den Fingern oder mit einem Pinsel in bunte Farbe getaucht. Hierbei ist anzuraten, die Grundfarben (Gelb, Blau und Rot) zu nutzen, da sich diese Farben gut mit den anderen Farben mischen lassen und neue Farben auf dem Endprodukt entstehen. In den 
Schuhkartondeckel kommt nun ein Blatt Papier und auf dieses Papier werden die mit Farbe bestrichenen Murmeln gelegt. Die Teilnehmenden bringen jetzt mit ihren Händen den Karton in Bewegung, sodass die Murmeln auf dem ganzen Blatt Farbspuren hinterlassen.

Nachdem jede Person ihr ganzes Bild voller Farbe hat, werden die »Kunstwerke» betrachtet. Mögliche Fragen sind:

- An was erinnert Dich/Sie Dein/lhr Kunstwerk?

- Kennt Ihr/kennen Sie Kunstwerke, die ähnlich aussehen?

- Welche Farben erblickst Du/erblicken Sie?

Interessant für die Teilnehmenden ist, dass neue Farben entstanden sind und sich Farbmischungen wie beispielsweise Grün, Lila oder Orange ergeben haben. Nach der Betrachtung und der Besprechung werden die Kunstwerke an einer sicheren Stelle gelagert, damit sie gut trocken können. Nachdem die Teilnehmenden mit ihrem Kunstwerk fertig sind, können Bilder von Jackson Pollock betrachtet werden, die auf eine ähnliche Art entstanden sind.

\section{Schwungtuch-Spiele}

Das Schwungtuch als Medium eignet sich gut, um für Jung und Alt Bewegungs- und Spielanlässe zu schaffen. Im folgenden Teil werden unterschiedliche Spiele mit dem Schwungtuch beschrieben.
- Die beiden Generationen stellen mit dem Schwungtuch unterschiedlich starke Wellen gemeinsam her.

- Ein Kind liegt oder sitzt auf dem Schwungtuch und die anderen bringen das Schwungtuch in Bewegung. Dabei wird das Kind gefragt, wie stark die Wellen sein dürfen. Als Variation darf ein/e Seniorln unter dem Schwungtuch sitzen und die anderen erzeugen die Wellen.

- Ein weicher Gegenstand (z.B. Schaumstoffball oder Kissen) kommt auf das Schwungtuch. Ziel ist es, gemeinsam das Schwungtuch so zu bewegen, dass der Gegenstand bis zur Decke fliegt und wieder zurück auf das Tuch fällt.

\section{Spiele mit Bällen}

Ballspiele sind den meisten SeniorInnen gut bekannt und vermitteln Sicherheit. Viele Herren aus der Gruppe spielten in ihrer Kindheit selbst Ballsportarten (z. B. Fußball, Handball oder Ball über die Schnur) und bekommen eine ganz besondere Motivation, wenn ein Ball ins Spiel kommt. Die Damen erzählen, dass sie früher nicht Fußball spielen durften und genießen es heute.

Bevor die psychomotorische Fachkraft neue Spiele einführt, sollten weiche Bälle im Stuhlkreis frei hin und her geworfen werden, da so mögliche Ängste vor Bällen abgebaut werden.

- Zwei unterschiedlich große Bälle werden schnell nach rechts weitergegeben. Dabei soll der kleinere den größeren Ball fangen (überho- len). Erklingt das Wort »Wechsel«, fängt der größere den kleineren Ball. Wenn ein Ball vom anderen Ball gefangen wurde, wird eine neue Runde eingeläutet.

- Eine Person (anfangs der Psychomotoriker) versucht, in der Mitte stehend oder sitzend, den Ball zu hindern zu einer anderen Person zu kommen. Wenn dies der Person in der Mitte gut gelingt, kann ein zweiter Ball dazukommen.

- Sitzfußball: Hier können selbst Menschen mit Beeinträchtigungen ins Fußballspiel integriert werden. Die Seniorlnnen schießen im Sitzen einen Softball hin und her. Ein bis zwei Kinder versuchen, ins Spiel zu kommen und den Ball zu anderen zu spielen.

\section{(10国) Literatur}

Eisenburger, M. (2012): Zuerst muss die Seele bewegt werden...: Ein theoriegeleitetes Praxisbuch. Verlag modernes lernen, Dortmund

Philippi-Eisenburger, M. (1991): Bewegungsarbeit mit älteren und alten Menschen. Theorie und Praxis der Motogeragogik. Hofmann Karl GmbH + Co, Schorndorf

Philippi-Eisenburger, M. (1990): Bewegungsarbeit mit älteren und alten Menschen. Hofmann Karl GmbH + Co, Schorndorf

Schmidt, D., Schießl, D. (1999): Ganzheitliches Gehirntraining für Senioren. Bildungsverlag Eins, Troisdorf

Frank Francesco Birk

DOI 10.2378/motorik2016.art17d 